\title{
MicroRNA-15a-5p inhibits endometrial carcinoma proliferation, invasion and migration via downregulation of VEGFA and inhibition of the Wnt/ $\beta$-catenin signaling pathway
}

\author{
HONGGANG WANG ${ }^{1}$, QINGJU YANG ${ }^{2},{\text { JIEPING } \mathrm{LI}^{3} \text {, WENPING CHEN }}^{4}, \mathrm{XIAO} \mathrm{JIN}^{5}$ and YAOWEN WANG \\ ${ }^{1}$ Department of Clinical Laboratory, Weifang People's Hospital, Weifang, Shandong 261041; \\ ${ }^{2}$ Department of Gynaecology, Linyi People's Hospital, Dezhou, Shandong 251500; \\ ${ }^{3}$ Department of Anesthesiology, Qingdao Hospital of Traditional Chinese Medicine, Qingdao University, \\ Qingdao, Shandong 266033; Departments of ${ }^{4}$ Cardiothoracic Surgery and ${ }^{5}$ Rehabilitation Medicine, \\ The People's Hospital of Zhangqiu Area, Jinan, Shandong 250200, P.R. China
}

Received January 16, 2020; Accepted December 16, 2020

DOI: $10.3892 / \mathrm{ol} .2021 .12570$

\begin{abstract}
Endometrial carcinoma (EC) is one of the most common malignant gynecological tumors. Dysregulation of microRNAs (miRNAs/miRs) is frequently identified in human tumors, playing key regulatory roles in tumor growth and metastasis. The present study aimed to explore the functions and potential mechanisms of miR-15a-5p in EC progression. RT-qPCR was used to detect the expression levels of miR-15a-5p and vascular endothelial growth factor A (VEGFA) mRNA. Western blot analysis was performed to examine the expression of related proteins. Functional assays, including proliferation and Transwell assays were performed to determine the roles of miR-15a-5p in EC progression. TargetScan and luciferase reporter assays were used to explore the potential target genes of miR-15a-5p. The results revealed that miR-15a-5p was underexpressed in EC tissue samples in comparison with that in matched normal tissue samples. The expression level of miR-15a-5p was associated with the clinicopathologic characteristics of EC patients. Notably, both in vitro and in vivo assays revealed that miR-15a-5p upregulation significantly inhibited EC growth and metastasis. Furthermore, bioinformatics analysis and dual luciferase reporter assay indicated that VEGFA was a candidate target of miR-15a-5p. Mechanistic investigation revealed that miR-15a-5p inhibited EC development via regulation of Wnt/ $\beta$-catenin pathway and targeting of VEGFA. In summary, the present results demonstrated that miR-15a-5p
\end{abstract}

Correspondence to: Dr Yaowen Wang, Department of Clinical Laboratory, Weifang People's Hospital, 151 Guangwen, Kuiwen, Weifang, Shandong 261041, P.R. China

E-mail: zhubrhvm67478@163.com

Key words: endometrial carcinoma, microRNA-15a-5p, vascular endothelial growth factor $\mathrm{A}, \mathrm{Wnt} / \beta$-catenin, epithelial-mesenchymal transition could inhibit EC development and may serve as a promising therapeutic biomarker in EC.

\section{Introduction}

Endometrial carcinoma (EC) originates from the endometrium and is ranked as the most prevalent reproductive malignancy (1). EC patients are mostly diagnosed in early clinical stages, but late-stage EC is frequently diagnosed with invasive metastasis and a high relapse rate (2). Therefore, the prognosis is usually poor with the occurrence of metastases and recurrence (3). To date, common treatments for EC mainly include radiation, chemotherapy, hormonal therapy, and surgeries with bilateral salpingo-oophorectomy and hysterectomy. These methods remain mainstream therapies in EC treatment and are generally only effective in patients at early stages of this disease (4). Therefore, it is urgent to elucidate the mechanism which occurs in EC pathogenesis for the identification of promising therapeutic strategies.

MicroRNA (miRNA/miR) is a type of small non-coding RNA (18-24 nucleotides in length) that could silence the target genes via inhibition of translation or degradation of mRNAs (5). A growing body of studies has revealed that miRs participate in regulating a variety of cellular processes, including metastasis, metabolism, differentiation and growth (6-8). Increasing evidence has revealed that aberrant miR expression is correlated with the genesis and development of multiple tumors, serving as either tumor suppressors or oncogenes. Evidence from Zhang et al revealed that miR-1299 was a tumor suppressor in prostate carcinoma, inhibiting cell growth and metastasis via regulation of never in mitosis gene A-related kinase 2 (NEK2) (9). Peng et al revealed that miR-31-5p enhanced colorectal carcinoma cell proliferation and metastasis by targeting NUMB (10). Zhen et al reported that miR-524 induced thyroid cancer cell apoptosis and suppressed cell proliferation through sperm-associated antigen 9 (SPAG9) (11). Moreover, emerging evidence has revealed that multiple miRs are implicated in EC. miR-486-5p could promote EC cell proliferation, migration, and invasion by targeting MARK1 (12); miR-101-3p 
induced autophagy in EC cells by targeting EZH2 (13); miR-522 facilitated the proliferation, invasion and migration of EC cells by directly binding to monoamine oxidase B (14). However, the roles of miR-15a-5p in EC have rarely been clarified. The present study aimed to elucidate the functions of miR-15a-5p in EC progression. Vascular endothelial growth factor (VEGF) plays crucial roles in tumor angiogenesis and is correlated with increased tumor metastasis and relapse (15). VEGFA is a potent angiogenic inducer of the VEGF family and increased VEGFA expression has been detected in various human tumors $(16,17)$. Recent studies have revealed that VEGFA may enhance cancer growth and progression in preclinical hepatocellular carcinoma models and genomic VEGFA amplification was identified as a predictive marker for hepatocellular carcinoma $(18,19)$. Overexpressed VEGFA was detected in colorectal cancer tissues and upregulation of VEGFA reversed the suppressive roles mediated by miR-150-5p overexpression in colorectal cancer (20). In EC, VEGF was indicated to function as a prognostic marker (21). miR-34a-5p downregulated VEGFA in endometrial stem cells, contributing to the pathogenesis of endometriosis (22). In the present study, the correlation between miR-15a-5p and VEGFA in EC progression was investigated.

\section{Materials and methods}

Cell culture and transfection. Human EC cell lines (AN3CA, HEC-1B, HEC-1A, and RL95-2) and endometrial stromal cell line T-HESCs were maintained in Dulbecco's modified Eagle's medium (DMEM) supplemented with $10 \%$ fetal bovine serum (FBS; both Gibco; Thermo Fisher Scientific, Inc.) in a humidified incubator of $5 \% \mathrm{CO}_{2}$ at $37^{\circ} \mathrm{C}$.

miR-15a-5p mimic, inhibitor and negative controls were constructed by Shanghai GenePharma Co., Ltd. miR-15a-5p mimics $(50 \mathrm{nM})$ were transfected into HEC-1A cells and miR-15a-5p inhibitor (50 nM) was transfected into AN3CA cells in accordance with the instructions of Lipofectamine 2000 (Invitrogen; Thermo Fisher Scientific, Inc.) at room temperature for $48 \mathrm{~h}$. The primer sequences were as follows: miR-15a-5p mimic (sense, 5'-UAGCAGCACAUAAUGGUU UGUG-3' and antisense, 5'-CAAACCAUUAUGUGCUGC UAUU-3'); mimic control (sense, 5'-UUCUCCGAACGU GUCACGUTT-3' and antisense, 5'-ACGUGACACGUUCGG AGAATT-3'); miR-15a-5p inhibitor (5'-CACAAACCAUUA UGUGCUGCUA-3'); inhibitor control (5'-CAGUACUUU UGUGUAGUACAA-3'). The cells were inoculated in a 6-well plate with an inoculation density of $\sim 2.5 \times 10^{6}$ cells/well, and incubated in a $37^{\circ} \mathrm{C}$ incubator. Logarithmically growing cells were selected and inoculated in a culture plate, and the cell confluence was $\sim 80 \%$ before transfection. Subsequent experimentations were performed at 48-h after transfection.

Clinical specimens. A total of 49 pairs of EC tissue samples and adjacent para-tumor tissues were acquired from the Weifang People's Hospital (Weifang, China) from August 2015 to June 2018. The age range of patients was 43-68 years (mean age, 51 years old). The inclusion criteria used were as follows: i) The EC tissue specimens were confirmed by pathology, and the adjacent para-carcinoma tissue specimens were confirmed by pathology that there was no tumor cell invasion; ii) the patients had not received immunotherapy, radiotherapy and chemotherapy or other antitumor treatments before surgery. The exclusion criteria were as follows: Patients who also had other i) malignant tumors; ii) severe cardiovascular and cerebrovascular diseases; and iii) hematological diseases. The tissue specimens were snap-frozen in liquid nitrogen after surgical resection and reserved at $-80^{\circ} \mathrm{C}$ for further usage. In the present study, no patient had received prior irradiation or chemotherapy. Informed consent was obtained from the subjects for the use of their samples for experimentation. The present study conformed to the Code of Ethics from the Ethics Committee of Weifang People's Hospital.

Reverse transcription-quantitative polymerase chain reaction $(R T-q P C R)$. Total RNA was isolated from EC/normal tissues and cells by TRIzol reagent (Invitrogen; Thermo Fisher Scientific, Inc.). To detect miR-15a-5p expression, TaqMan MicroRNA Reverse Transcription Kit (Thermo Fisher Scientific, Inc.) was used to reverse transcribe total RNA into cDNA. For VEGFA detection, cDNA was synthesized by Prime Script RT Reagent Kit (Takara Biotechnology Co., Ltd.). Thereafter, qPCR was conducted on an ABI 7500 Detection System with SYBR Premix Ex Taq ${ }^{\mathrm{TM}}$ kit (Takara Biotechnology Co., Ltd.) or a TaqMan MicroRNA Assay Kit (Thermo Fisher Scientific, Inc.) for VEGFA or miR-15a-5p, respectively. The thermocycling conditions were as follows: Pre-denaturation at $94^{\circ} \mathrm{C}$ for $5 \mathrm{~min}$, followed by 40 cycles of denaturation at $94^{\circ} \mathrm{C}$ for $30 \mathrm{sec}$, annealing at $55^{\circ} \mathrm{C}$ for $30 \mathrm{sec}$ and extension at $72^{\circ} \mathrm{C}$ for $90 \mathrm{sec}$. The relative expression levels were analyzed using the $2^{-\Delta \Delta \mathrm{Cq}}$ method (23), with normalization to GAPDH or U6. The primers are presented in Table I.

3-(4,5-Dimethylthiazol-2-yl)-2,5-diphenyl tetrazolium bromide (MTT) assay. Transfected EC cells $\left(5 \times 10^{3}\right)$ were seeded into 96 -well plates. Then, cell viability was assessed at indicated time-points (0, 24, 48 and $72 \mathrm{~h})$ after transfection by MTT assay. In brief, $10 \mu \mathrm{l}$ MTT solution $(0.5 \mathrm{mg} / \mathrm{ml}$; Sigma-Aldrich; Merck KGaA) was added into the cells, followed by incubation in a humidified chamber at $37^{\circ} \mathrm{C}$ for $4 \mathrm{~h}$. Subsequently, $150 \mu \mathrm{l}$ DMSO (Sigma-Aldrich; Merck $\mathrm{KGaA}$ ) was added to each well for full dissolution of crystallization. The absorbance at $490 \mathrm{~nm}$ was detected by a microplate reader (BioTek Instruments, Inc.).

Transwell assay. The influence of miR-15a-5p on EC cell invasion and migration was assessed by Transwell chamber with an $8-\mu \mathrm{m}$ pore size membrane (Corning, Inc.). For invasion assays, cells in FBS-free medium were added to the upper Matrigel-coated Transwell chamber. For the migration assay, the chamber was not coated with Matrigel. In addition, the medium containing $10 \%$ FBS was added into the bottom chamber. The cells were cultured at $37^{\circ} \mathrm{C}$ for $48 \mathrm{~h}$. The migrated/invasive cells were fixed with $95 \%$ ethyl alcohol for $15 \mathrm{~min}$ at room temperature and stained with $0.1 \%$ crystal violet for $10 \mathrm{~min}$ at room temperature. Finally, the stained cells were quantified under a light microscope (BX-42; Olympus Corporation; magnification, x200).

Western blot analysis. Total protein lysate was obtained by ice-cold radioimmunoprecipitation lysis buffer (RIPA; Thermo Fisher Scientific, Inc.), and quantified with a 
Table I. Primer sequences for RT-qPCR.

\begin{tabular}{ll}
\hline Primer & \multicolumn{1}{c}{ Sequence } \\
\hline miR-15a-5p & F: 5'-TAAGGCACGCGGTGAATGCC-3' \\
& R: 5'-GCCTGGGTCTCACCATGTAG-3' \\
U6 & F: 5'-CTCGCTTCGGCAGCACA-3' \\
& R: 5'-AACGCTTCACGAATTTGCGT-3' \\
VEGFA & F: 5'-TGGCTCACTGGCTTGCTCTA-3' \\
& R: 5'-ATCCAACTGCACCGTCACAG-3' \\
GAPDH & F: 5'-CCAGGTGGTCTCCTCTGACTT-3' \\
& R: 5'-GTTGCTGTAGCCAAATTCGTTGT-3'
\end{tabular}

miR-15a-5p, microRNA-15a-5p; F, forward; R, reverse; U6, small nuclear RNA (snRNA); VEGFA, vascular endothelial growth factor A; GAPDH, glyceraldehyde-3-phosphate dehydrogenase.

bicinchoninic acid (BCA) assay kit (Pierce; Thermo Fisher Scientific, Inc.). Then, $30 \mu \mathrm{g}$ lysate was separated by $10 \%$ sodium dodecyl sulphate-polyacrylamide gel electrophoresis (SDS-PAGE), and transferred onto a polyvinylidene fluoride (PVDF) membrane. The membrane was blocked with 5\% skim milk at room temperature for $1 \mathrm{~h}$ in Tris-Buffered Saline and $0.1 \%$ Tween-20 (TBST). Thereafter, the membranes were incubated overnight at $4^{\circ} \mathrm{C}$ with a primary antibody, followed by a 2-h incubation with secondary antibody goat anti-rabbit IgG H\&L (HRP) (1:4,000; product code ab7090) at room temperature. The primary antibodies were as follows: Antibodies against VEGFA (1:10,000; product code ab52917), cyclin D1 (1:1,000; product code ab40754), c-Myc (1:1,000; product code ab32072), $\beta$-catenin (1:1,000; product code ab68183), p-GSK3 $\beta$ (1:1,000; product code ab131097), total GSK3 $\beta$ (1:1,000; product code ab227208), E-cadherin (1:2,000; product code ab133597), N-cadherin (1:2,000; product code ab207608), vimentin (1:1,000; product code ab137321) and GAPDH (1:1,000; product code ab128915). All antibodies were obtained from Abcam. GAPDH served as an internal control. Finally, the membranes were visualized with the ECL reagent (EMD Millipore). ImageJ software (version 1.48; National Institutes of Health) was used for densitometry.

Bioinformatic analysis. The putative human target genes of miR-15a-5p were analyzed using the TargetScan (version 6.0; targetscan.org/) (24,25).

Luciferase reporter assay. Two types of VEGFA 3'UTR fragments [wild-type (WT) or mutant (MUT)] were inserted into the pGL3 vector (Promega Corporation) to obtain the VEGFA 3'UTR-WT or -MUT reporter. EC cells were co-transfected with miR-15a-5p mimics and VEGFA 3'UTR-WT or -MUT by Lipofectamine 2000 (Invitrogen; Thermo Fisher Scientific, Inc.). After $48 \mathrm{~h}$ of transfection, the Dual-Luciferase Reporter Assay System (Promega Corporation) was applied to evaluate the luciferase activity. Relative firefly luciferase activity was normalized to Renilla luciferase activity.

In vivo tumor xenograft mouse model. The lentiviral plasmid containing miR-15a-5p (lenti-miR-15a-5p) or a control (lenti-control) were constructed and synthesized by Shanghai GenePharma Co., Ltd. A three-plasmid system and $9 \mu \mathrm{g}$ lentiviral vectors were co-transfected into the 293 cells (Invitrogen; Thermo Fisher Scientific, Inc.) through Lipofectamine ${ }^{\circledR} 2000$ (Invitrogen; Thermo Fisher Scientific, Inc.). The rate of lentiviral plasmid: Packaging vector: Envelope was 1:1:1. Lentivirus-containing medium was collected $48 \mathrm{~h}$ after transfection and used to infect HEC-1A cells $\left(5 \times 10^{6}\right.$ cells/well, in six-well plates) at a multiplicity of infection (MOI) of 20. Subsequently, $48 \mathrm{~h}$ after lentiviral infection, $1 \mu \mathrm{g} / \mathrm{ml}$ puromycin was used to select the infected cells. After $72 \mathrm{~h}$, transfected cells were suspended in DMEM and injected subcutaneously into the dorsal flank of the nude mice. For in vivo xenograft studies, a total of $12 \mathrm{BALB} / \mathrm{c}$ nude mice (aged 4-6 weeks; female; 20-25 g) were obtained from Beijing Vital River Laboratory Animal Technology Co., Ltd. and the animal experiments were approved by the Animal Care and Use Committee of Weifang People's Hospital. The nude mice were housed in pathogen-free environment with a 12 -h light/dark cycle, temperature $\left(26^{\circ} \mathrm{C}\right)$, humidity $(50 \%)$ and free access to food and water. HEC-1A cells $\left(5 \times 10^{6}\right)$ packaged with lentiviral vector containing miR-15a-5p (lenti-miR-15a-5p) or a control (lenti-control) were suspended in DMEM and injected subcutaneously into the dorsal flank of the nude mice ( $n=6$ per group). Then, 10 days after inoculation, tumor volumes were measured every 3 days following the formula: Volume $\left(\mathrm{mm}^{3}\right)=\left(\right.$ length $\mathrm{x}$ width $\left.{ }^{2}\right) / 2$. Subsequently, the mice were sacrificed by cervical dislocation after 28 days. All surgical procedures were performed under $1 \%$ sodium pentobarbital anesthesia $70 \mathrm{mg} / \mathrm{kg}$, and all efforts were made to minimize suffering.

Statistical analysis. All experiments were conducted at least thrice. Statistical analyses were performed by Statistical Product and Service Solutions (SPSS) software version 17.0 (SPSS Inc.). Comparisons of two or more groups were determined by unpaired Student's t-test or one-way ANOVA analysis along with Dunnett's post hoc test. The Kaplan-Meier analysis along with log-rank test was applied to analyze the overall survival (OS) of EC patients. Comparison of the tumor volume between the two groups was performed by unpaired t-test. Statistical analysis for miR-15a-5p expression and the clinicopathological characteristics was performed by the $\chi^{2}$ test. $\mathrm{P}<0.05$ indicated a statistically significant difference.

\section{Results}

Downregulated miR-15a-5p is associated with unfavorable prognosis in EC patients. To explore the functional roles of miR-15a-5p in EC, miR-15a-5p levels in EC tissues and cell lines were detected. According to RT-qPCR, it was revealed that miR-15a-5p expression in EC tissues was lower than that in the matched normal tissue samples (Fig. 1A). Similarly, compared to T-HESCs, EC cells had significantly lower miR-15a-5p expression (Fig. 1B). Moreover, to investigate the clinicopathological significance of miR-15a-5p in EC progression, the EC patients were grouped into a high-miR-15a-5p expression group and a low-miR-15a-5p expression group based on the median miR-15a-5p expression level. As revealed in Table II, patients in the low-miR-15a-5p expression group 
Table II. Association of miR-15a-5p expression with the clinicopathological characteristics of EC patients.

\begin{tabular}{|c|c|c|c|c|}
\hline \multirow[b]{2}{*}{ Clinicopathological Features } & \multirow[b]{2}{*}{ Cases $(n=49)$} & \multicolumn{2}{|c|}{ miR-15a-5 $p^{a}$ expression } & \multirow[b]{2}{*}{ P-value } \\
\hline & & $\operatorname{High}(\mathrm{n}=17)$ & Low $(n=32)$ & \\
\hline Age (years) & & & & 0.203 \\
\hline$>56$ & 26 & 7 & 19 & \\
\hline$\leq 56$ & 23 & 10 & 13 & \\
\hline Pathology classification & & & & 0.061 \\
\hline Well + moderate & 24 & 9 & 15 & \\
\hline Poor & 25 & 8 & 17 & \\
\hline FIGO stage & & & & $0.031^{\mathrm{b}}$ \\
\hline I-II & 22 & 13 & 9 & \\
\hline III-IV & 27 & 4 & 23 & \\
\hline Lymph-node metastasis & & & & $0.012^{\mathrm{b}}$ \\
\hline Yes & 29 & 5 & 24 & \\
\hline No & 20 & 12 & 8 & \\
\hline ER status & & & & 0.078 \\
\hline Positive & 20 & 9 & 11 & \\
\hline Negative & 39 & 8 & 21 & \\
\hline PR status & & & & 0.092 \\
\hline Positive & 23 & 10 & 13 & \\
\hline Negative & 26 & 7 & 19 & \\
\hline
\end{tabular}

${ }^{a}$ The mean expression level of miR-15a-5p was used as the cutoff. ${ }^{b}$ Statistically significant. miR-15a-5p, microRNA-15a-5p; EC, endometrial carcinoma; FIGO, International Federation of Gynecology and Obstetrics; ER, estrogen receptor; PR, progesterone receptor.
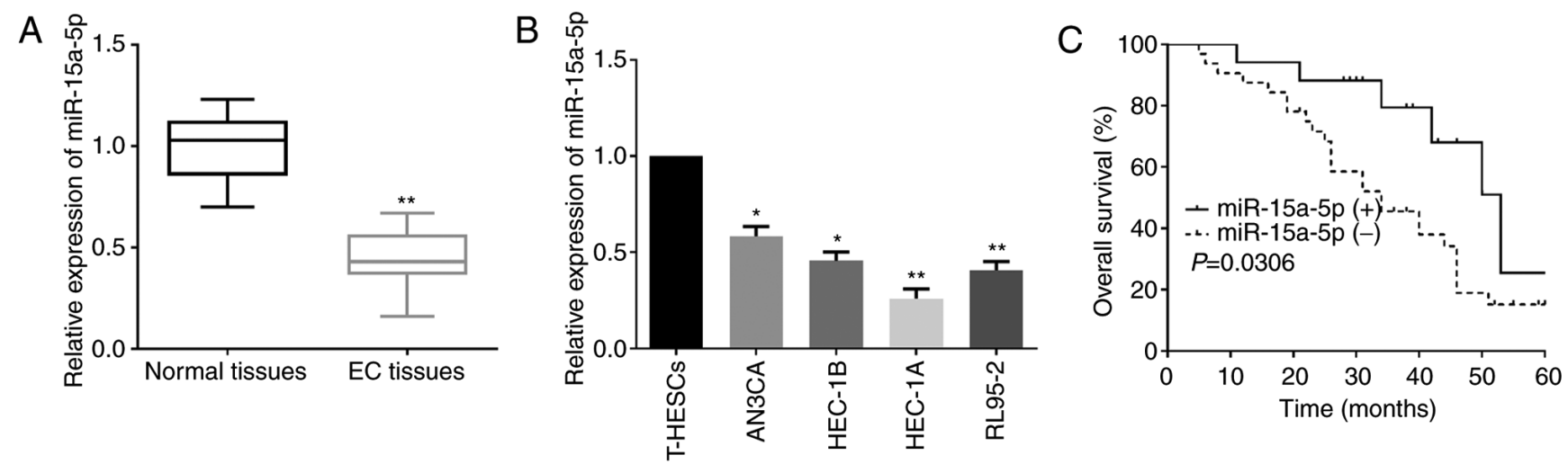

Figure 1. Decreased miR-15a-5p expression in EC is associated with aggressive clinicopathologic features. (A and B) RT-qPCR was performed to detect miR-15a-5p expression in EC tissues and cell lines. (C) Kaplan-Meier analysis revealed that low miR-15a-5p expression was significantly associated with shorter OS of EC patients. ${ }^{*} \mathrm{P}<0.05$ and ${ }^{* *} \mathrm{P}<0.01$. EC, endometrial carcinoma; RT-qPCR, reverse transcription-quantitative polymerase chain reaction; miR-15a-5p, microRNA-15a-5p; OS, overall survival.

exhibited aggressive clinicopathological phenotypes in comparison to patients in the high-miR-15a-5p expression group. Additionally, low miR-15a-5p expression was also associated with unfavorable prognosis of EC patients as demonstrated by Kaplan-Meier analysis (Fig. 1C).

miR-15a-5p suppresses EC cell viability. Since the decreased miR-15a-5p expression in EC was confirmed, functional assays were further carried out to determine its specific roles in EC. Firstly, miR-15a-5p mimics or inhibitor were transfected into HEC-1A and AN3CA cells according to their relatively low and high endogenous miR-15a-5p expression. As revealed in Fig. 2A and B, the successful overexpression or inhibition of miR-15a-5p was confirmed by RT-qPCR. Then, an MTT assay was performed to detect the proliferation ability of transfected cells. The results revealed that miR-15a-5p upregulation significantly suppressed HEC-1A cell proliferation (Fig. 2C). Conversely, the viability of AN3CA cells was significantly increased by miR-15a-5p inhibitor (Fig. 2D). 
A

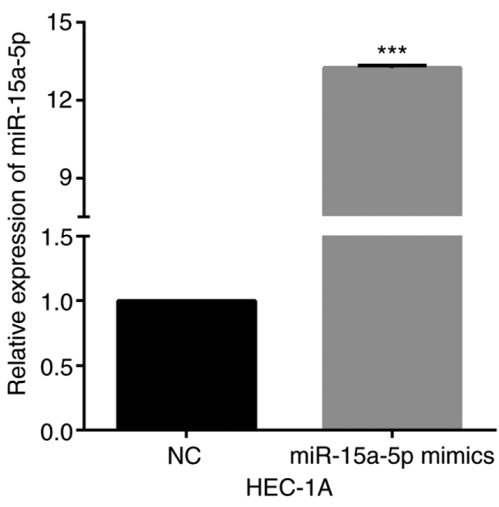

C

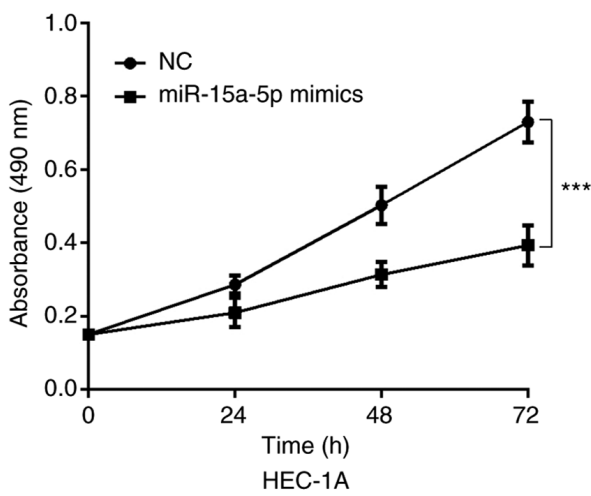

B
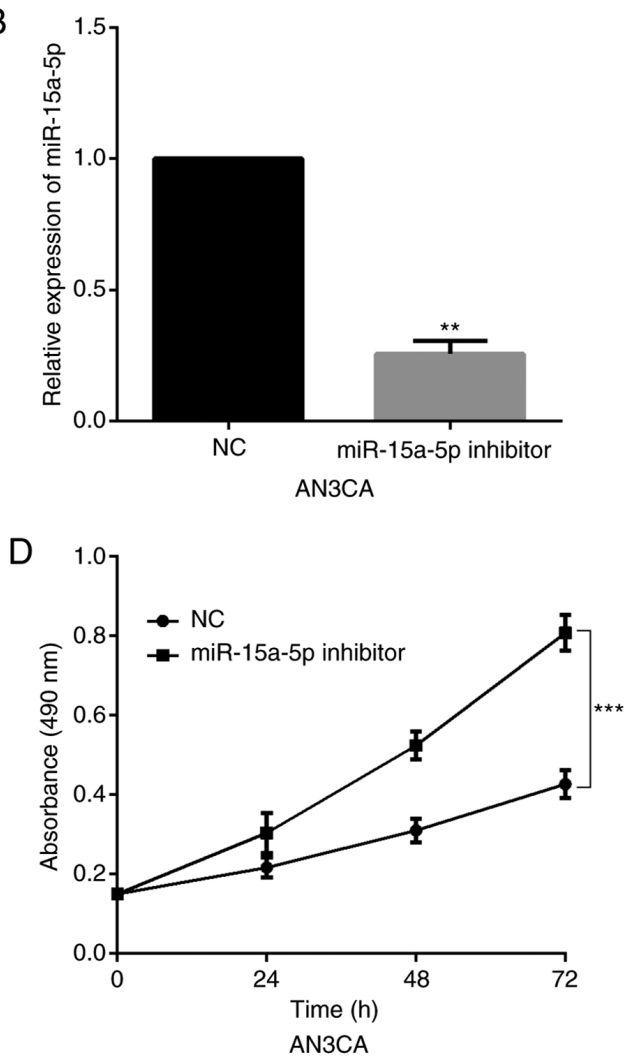

Figure 2. miR-15a-5p upregulation suppresses EC cell viability. (A and B) miR-15a-5p overexpression or inhibition in HEC-1A or AN3CA cells was confirmed by RT-qPCR. (C and D) Cell viability of HEC-1A or AN3CA cells which were transfected with miR-15a-5p mimics or inhibitor was detected by MTT assay. ${ }^{* *} \mathrm{P}<0.01$ and $^{* * * *} \mathrm{P}<0.001$. miR-15a-5p, microRNA-15a-5p; EC, endometrial carcinoma; RT-qPCR, reverse transcription-quantitative polymerase chain reaction; MTT, 3-(4,5-dimethylthiazol-2-yl)-2,5-diphenyl tetrazolium bromide; NC, negative control.

miR-15a-5p inhibits EC cell invasion and migration. Subsequently, Transwell assays were performed to explore the effects of miR-15a-5p on EC cell invasion and migration. In Fig. $3 \mathrm{~A}$ and $\mathrm{B}$, it was observed that both the invasion and migration capacities of HEC-1A cells were significantly impaired by miR-15a-5p mimics. Conversely, miR-15a-5p silencing in AN3CA cells significantly facilitated cell invasion and migration (Fig. 3C and D). In sum, all these data revealed that miR-15a-5p functioned as a tumor suppressor in EC.

miR-15a-5p directly targets and negatively regulates VEGFA. Mechanistically, the target genes of miR-15a-5p were identified by Targetscan, and the results revealed that VEGFA was a candidate target of miR-15a-5p. The targeting sites between VEGFA and miR-15a-5p are presented in Fig. 4A. Then, a luciferase reporter assay was conducted to verify the association. Findings revealed that the luciferase activity of VEGFA-3'UTR-WT was significantly decreased by miR-15a-5p mimics in EC cells, whereas no evident variation on luciferase activity of VEGFA-3'UTR-MUT was detected (Fig. 4B). Moreover, the regulatory roles of miR-15a-5p in VEGFA expression were examined by performing western blotting. miR-15a-5p silencing significantly enhanced VEGFA levels in AN3CA cells (Fig. 4C). In addition, VEGFA levels in the miR-15a-5p-overexpressed HEC-1A cells were significantly decreased (Fig. 4D). The aforementioned collective data revealed that VEGFA was a direct target of miR-15a-5p.
Upregulated VEGFA in EC indicates poor prognosis of EC patients. Since VEGFA was confirmed as a target of miR-15a-5p, the clinical value of VEGFA in EC development was further explored. As indicated by RT-qPCR, VEGFA was significantly upregulated in EC tissues when compared to that in the adjacent non-tumor tissue samples (Fig. 5A). Similarly, the increased VEGFA levels were also identified in EC cell lines (Fig. 5B). Subsequently, Kaplan-Meier analysis was performed to clarify the role of the ectopic VEGFA expression in EC patients. As revealed in Fig. 5C, high VEGFA expression resulted in a significantly poorer prognosis of EC patients than low VEGFA expression. Data revealed that VEGFA was partially involved in the functions of miR-15a-5p in EC.

miR-15a-5p regulates the Wnt/ $\beta$-catenin signaling pathway and epithelial-mesenchymal transition (EMT) in EC cells. The potential mechanism of miR-15a-5p was further investigated by western blot analysis. Firstly, the expression levels of EMT-related proteins were detected. In HEC-1A cells, miR-15a-5p mimics significantly increased the expression level of E-cadherin while significantly decreasing vimentin and $\mathrm{N}$-cadherin expression levels (Fig. 6A). In contrast, E-cadherin was downregulated whereas vimentin and $\mathrm{N}$-cadherin were upregulated by miR-15a-5p inhibitor in AN3CA cells (Fig. 6B). Expression levels of Wnt/ $\beta$-catenin-related proteins, including activated $\beta$-catenin, c-Myc, cyclin D1, and p-GSK3 $\beta$ were significantly inhibited by miR-15a-5p overexpression (Fig. 6C). Moreover, in AN3CA cells, miR-15a-5p inhibitor had 
A
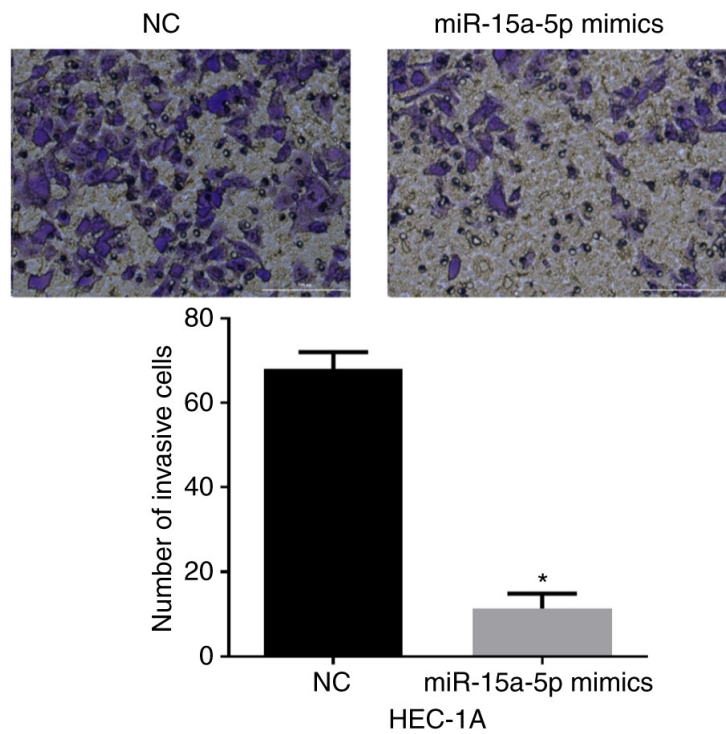

C
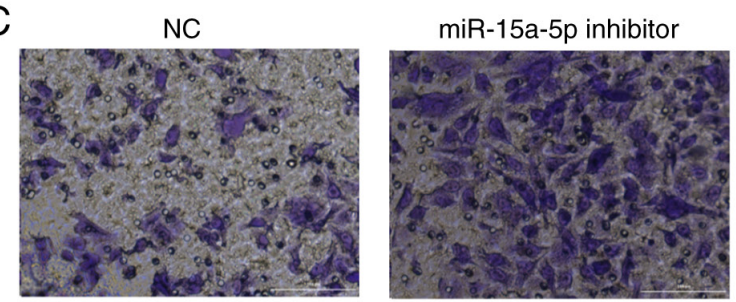

B
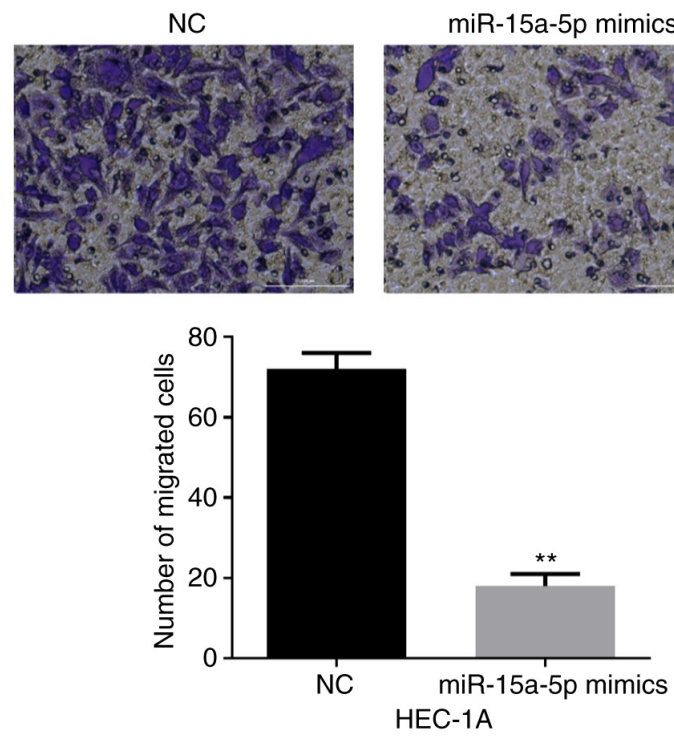

D

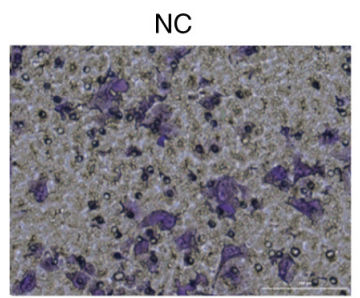

miR-15a-5p inhibitor

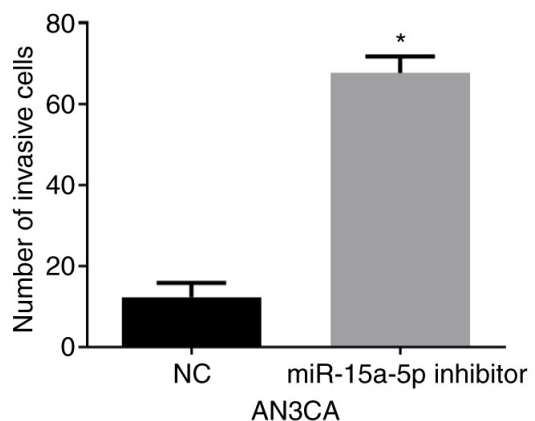

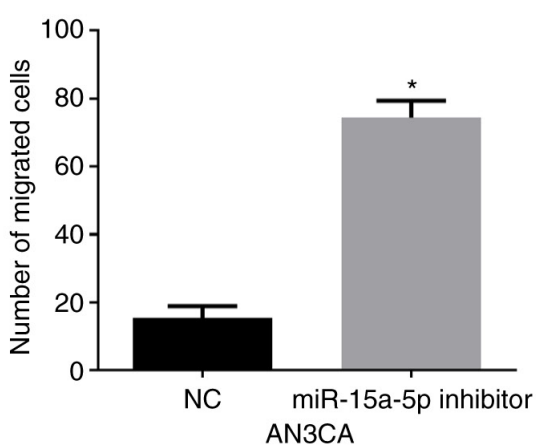

Figure 3. miR-15a-5p inhibits EC cell invasion and migration. (A and B) Transwell assays indicated that the invasion and migration abilities of HEC-1A cells were significantly suppressed by miR-15a-5p mimics. (C and D) The invasion and migration capacities of AN3CA cells were promoted by miR-15a-5p inhibitor as detected by Transwell assays. ${ }^{*} \mathrm{P}<0.05$ and ${ }^{* *} \mathrm{P}<0.01$. miR-15a-5p, microRNA-15a-5p; EC, endometrial carcinoma; NC, negative control.

the opposite effects on the Wnt/ $\beta$-catenin pathway (Fig. 6D). Collectively, miR-15a-5p could block EMT and Wnt/ß-catenin pathway of EC cells.

miR-15a-5p suppresses EC tumorigenesis in vivo. The effect of miR-15a-5p on EC in vivo was further investigated by tumorigenicity analysis in mice xenografts. Firstly, the expression of miR-15a-5p in HEC-1A cells, which were transfected with lenti-miR-15a-5p, was detected. As revealed in Fig. 7A, miR-15a-5p was stably upregulated in cells with transfection of lenti-miR-15a-5p. As revealed by a significant decrease in tumor volumes, miR-15a-5p overexpression markedly suppressed tumor growth in mice xenografts compared with controls (Fig. 7B and C). Moreover, the regulatory functions of miR-15a-5p in regulating VEGFA expression and the Wnt/ $\beta$-catenin pathway in an in vivo mouse model were confirmed by western blotting. The results revealed that the expression levels of VEGFA and Wnt/ $\beta$-catenin pathway-related genes were inhibited by miR-15a-5p (Fig. 7D).

\section{Discussion}

Generally, advanced EC patients usually have a high recurrence rate and poor prognosis, and existing therapeutic methods for EC patients have various shortcomings (26). In brief, chemotherapy seriously affects the quality of life, radiotherapy causes gastrointestinal reactions, ovarian resection induces menopausal symptoms in premenopausal women, and uterus removal leads to infertility (27). Moreover, they also severely affect the treatment and diagnosis progression of EC. Moreover, the molecular biology of EC genesis and progression remain indistinct. Hence, to explore the EC pathogenesis and identify novel diagnostic and therapeutic biomarkers for EC prognosis is of great significance. In recent years, 
A
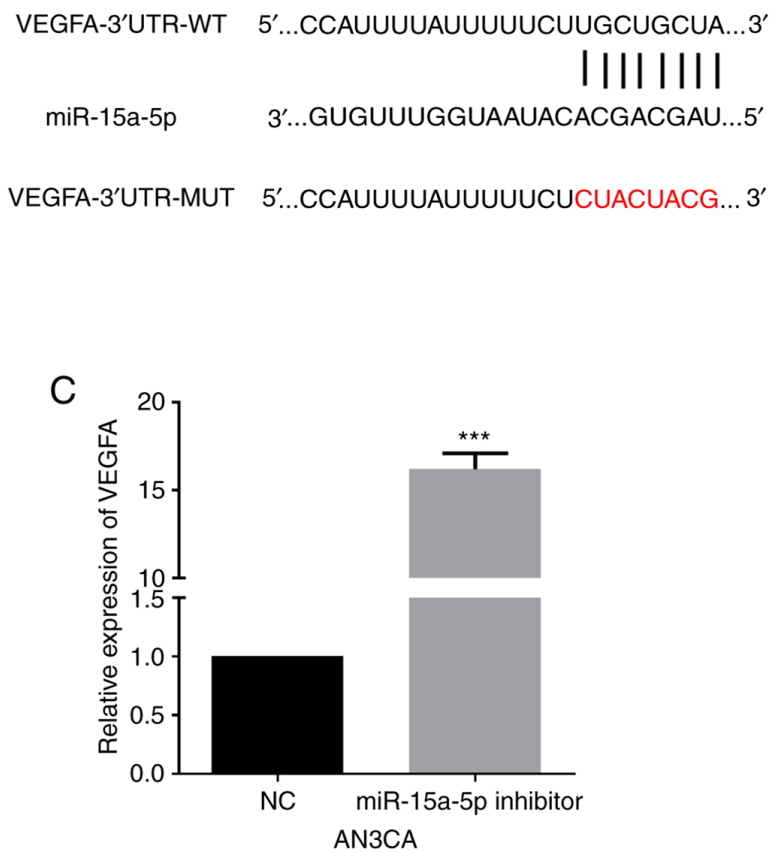

$\mathrm{B}$

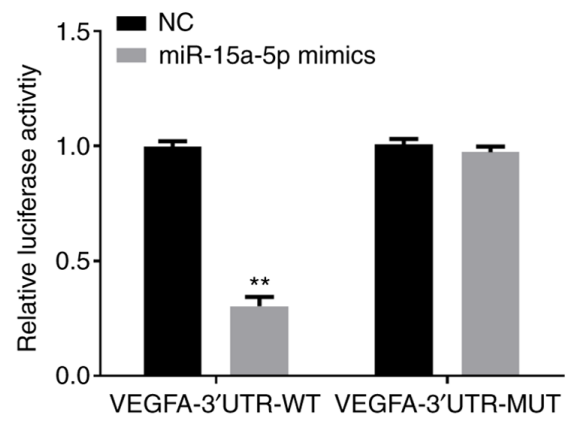

D

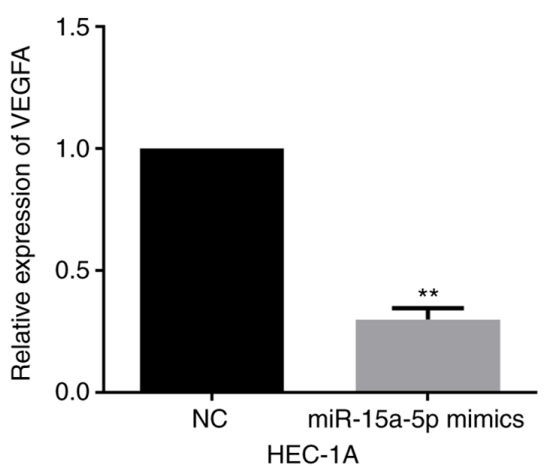

Figure 4. miR-15a-5p regulates VEGFA expression via targeting its 3'UTR. (A) Putative binding sequences of miR-15a-5p in VEGFA 3'-UTR are presented. (B) Luciferase reporter activity of VEGFA 3'-UTR-WT or -MUT vector in EC cells transfected with miR-15a-5p mimics was detected. (C and D) The influence of miR-15a-5p on VEGFA expression was examined by RT-qPCR in EC cells. ${ }^{* *} \mathrm{P}<0.01$ and ${ }^{* * *} \mathrm{P}<0.001$. miR-15a-5p, microRNA-15a-5p; VEGFA, vascular endothelial growth factor A; WT, wild-type; MUT, mutant; RT-qPCR, reverse transcription-quantitative polymerase chain reaction; EC, endometrial carcinoma; NC, negative control.

A

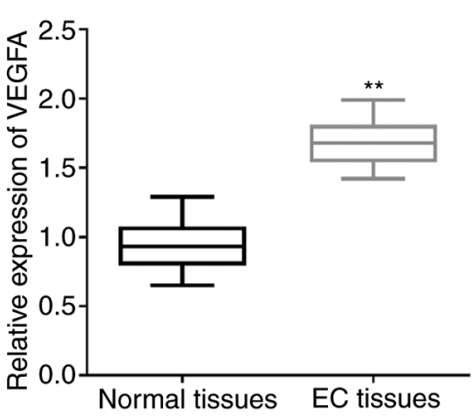

$\mathrm{B}$

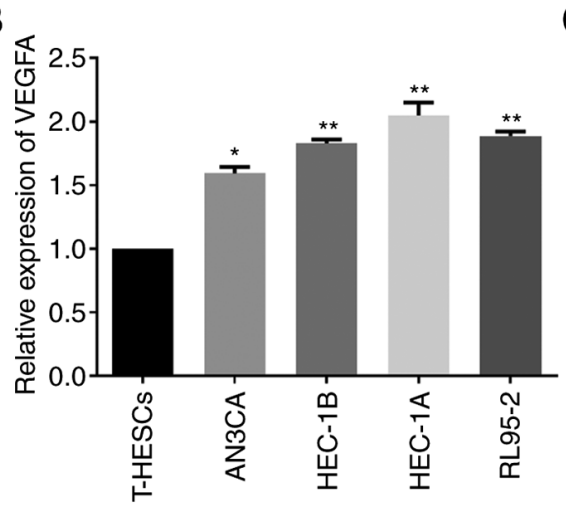

C

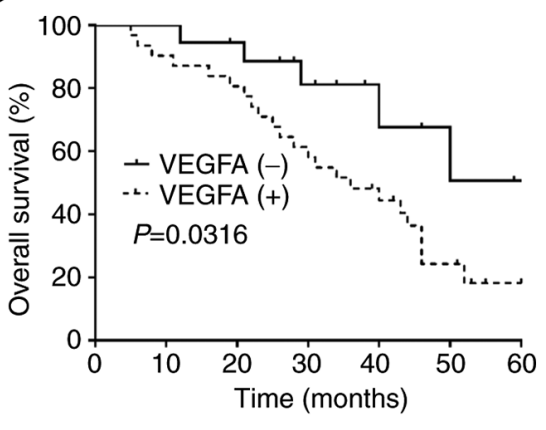

Figure 5. Prognostic significance of VEGFA in EC. (A and B) VEGFA expression levels were detected in EC tissues and cells by RT-qPCR. (C) The prognostic value of VEGFA in EC patients was analyzed by Kaplan-Meier analysis. " $\mathrm{P}<0.05$ and ${ }^{* *} \mathrm{P}<0.01$. EC patients were assigned into high (+) and low (-) VEGFA groups based on the median VEGFA expression level. VEGFA, vascular endothelial growth factor A; EC, endometrial carcinoma.

differentially expressed miRNAs have been identified in EC, indicating their importance in EC development (28).

Tumorigenesis progresses by losing adhesion and tight junctions as well as gaining invasive features, leading to EMT, which is characterized by suppression of E-cadherin expression and enhancement of $\mathrm{N}$-cadherin expression (29). EMT is a pivotal step in tumor invasion and metastasis, and the understanding of EMT has greatly improved owing to the identification of miRs, which regulate downstream events and signaling pathways (30). Furthermore, in EC progression, molecular events still require further exploration and miR-15a-5p may play important roles in EMT. As a critical developmental pathway, Wnt/ $\beta$-catenin signaling is considered important for cell differentiation, self-regeneration, growth, developmental decisions in tissue homeostasis, embryonic development, and tumorigenesis $(31,32)$. Abnormal activation of the Wnt/ $\beta$-catenin pathway may lead to the development of multiple human tumors (33). Previous studies have revealed that $\mathrm{Wnt} / \beta$-catenin is involved in EC progression $(34,35)$. For instance, the Wnt/ $\beta$-catenin pathway was involved in the functions of the hsa_circ_0002577/miR-197/CTNND1 axis in EC development (34). Moreover, a study by Chen et al revealed that miR-202 inhibited cell migration and invasion through targeting FGF2 and inactivating Wnt/ $\beta$-catenin signaling in 
A
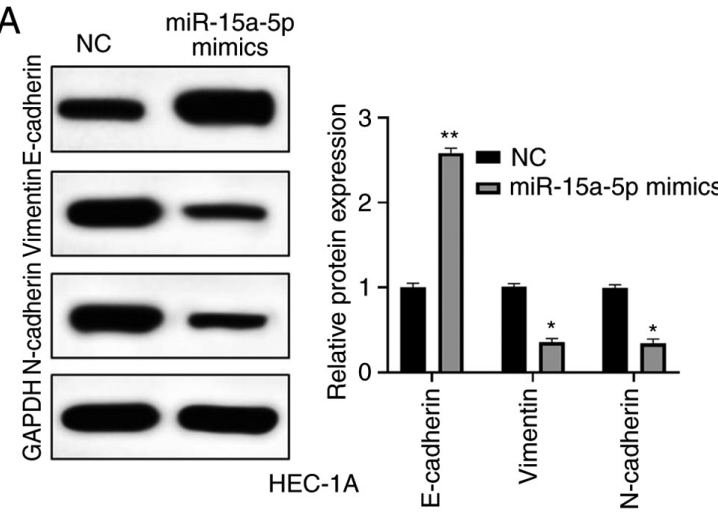

C

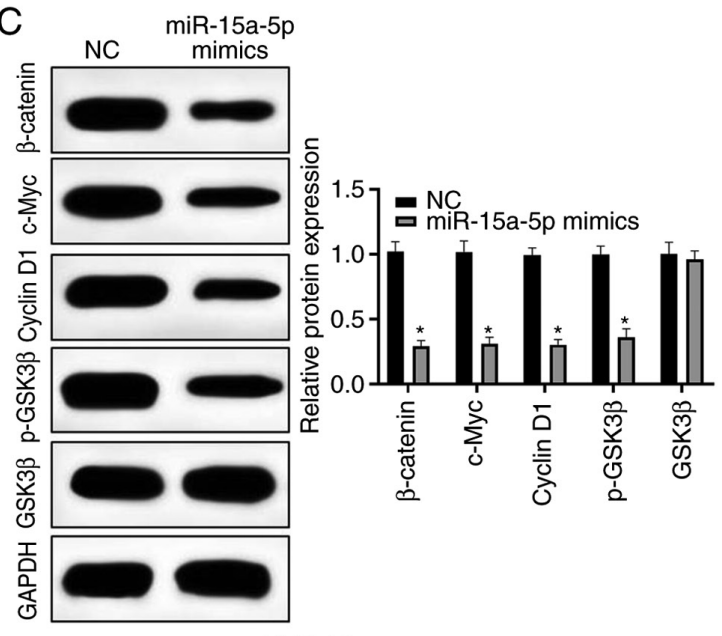

B

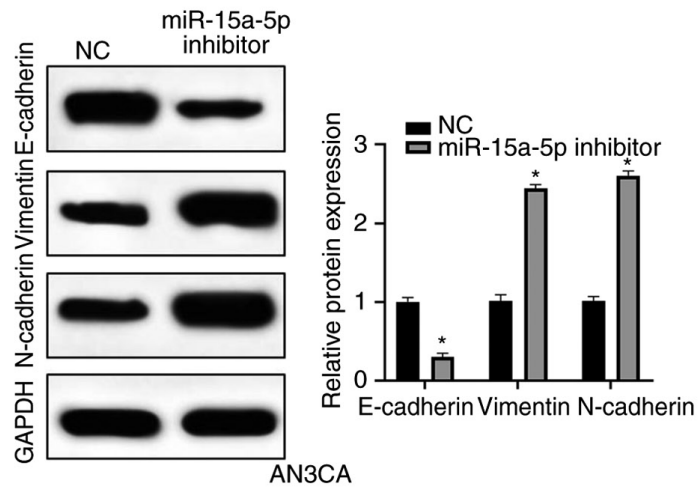

D

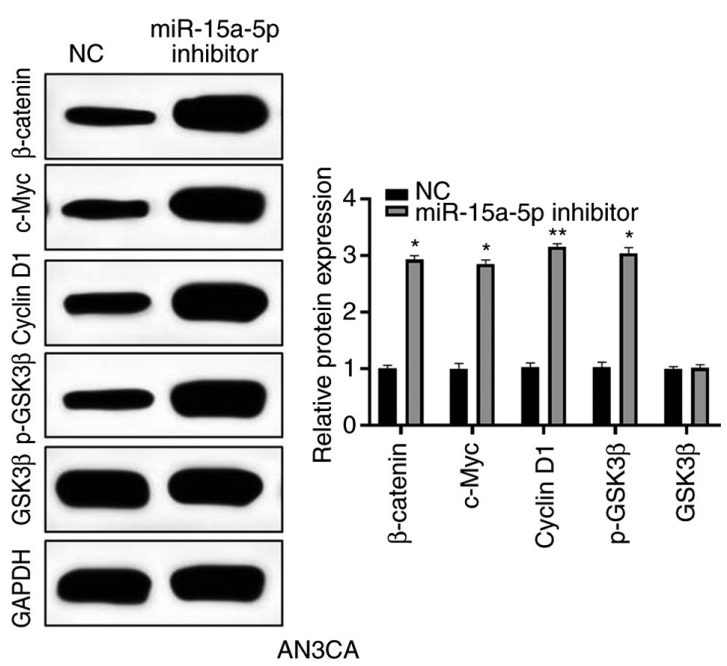

Figure 6. miR-15a-5p regulates EMT and the Wnt/ $\beta$-catenin pathway in EC cells. (A and B) miR-15a-5p regulated EC cell EMT as demonstrated by western blot analysis. (C and D) The functions of miR-15a-5p in EC cell Wnt $/ \beta$-catenin pathway were determined by western blot analysis. ${ }^{*} \mathrm{P}<0.05$ and ${ }^{* *} \mathrm{P}<0.01$. miR-15a-5p, microRNA-15a-5p; EMT, epithelial-mesenchymal transition; EC, endometrial carcinoma; NC, negative control.

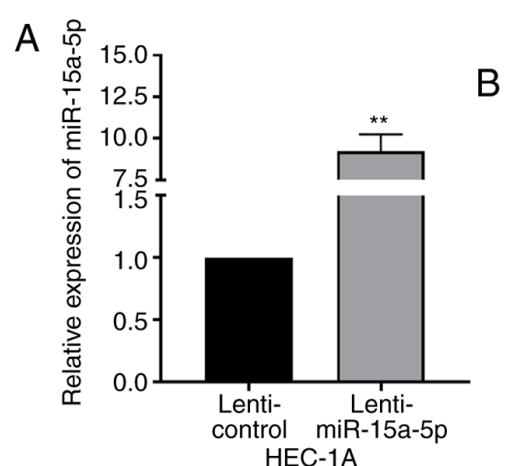

D

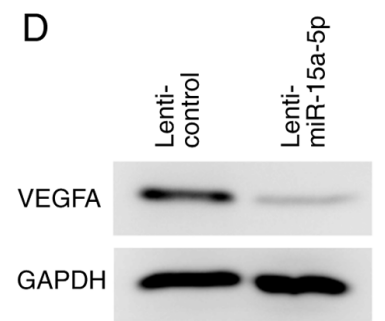

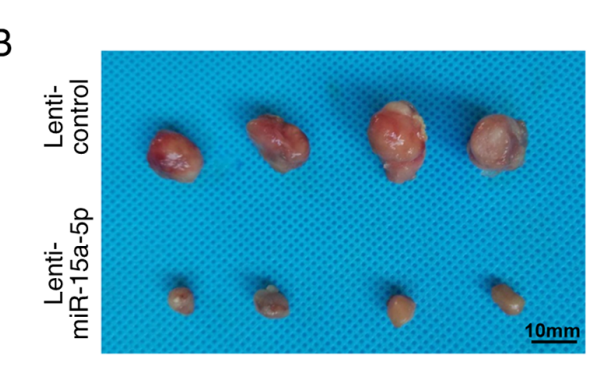

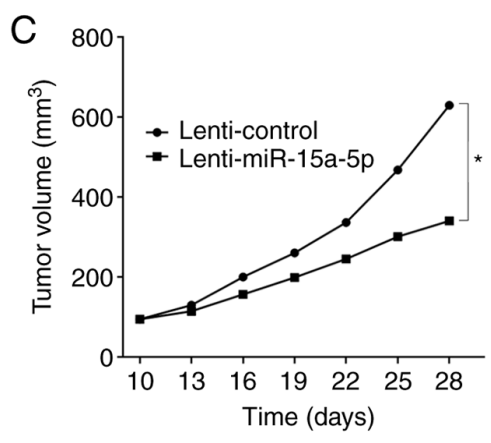

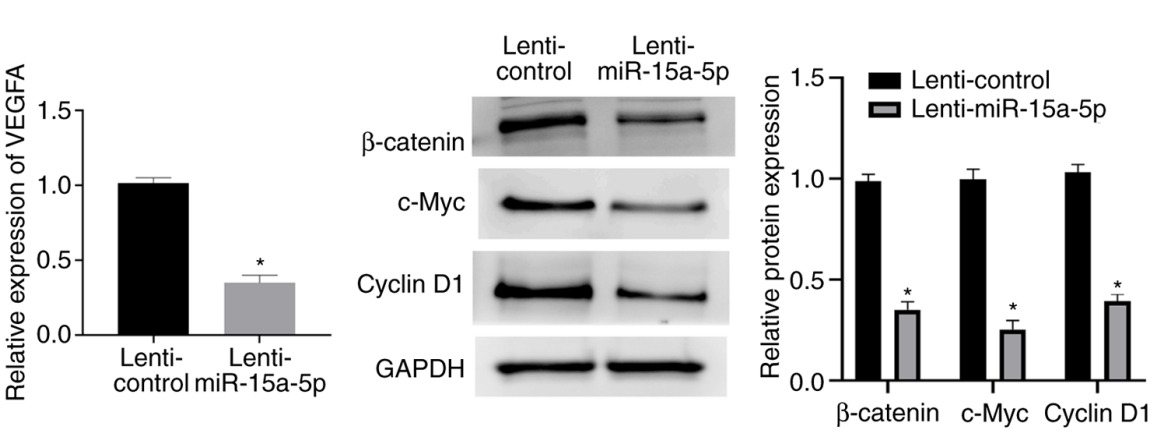

Figure 7. miR-15a-5p suppresses EC tumorigenesis in vivo. (A) The transfection efficiencies of lenti-miR-15a-5p in HEC-1A cells were detected by RT-qPCR (B) Representative tumor images of the xenograft models in different groups. (C) Tumor growth of xenograft models. (D) The functions of miR-15a-5p in VEGFA and Wnt/ $\beta$-catenin signaling in in vivo mouse models were confirmed by western blotting. ${ }^{*} \mathrm{P}<0.05$ and ${ }^{* *} \mathrm{P}<0.01$. miR-15a-5p, microRNA-15a-5p; EC, endometrial carcinoma; RT-qPCR, reverse transcription-quantitative polymerase chain reaction; NC, negative control. 
EC (36). In the present study, the functions of Wnt/ $\beta$-catenin signaling in EC progression regulated by miR-15a-5p were further explored. Accumulating studies have revealed that miR-15a-5p is a pivotal regulator in numerous human tumors. For instance, miR-15a-5p was identified as a prognostic predictor in recurrent colorectal adenocarcinoma (37). Moreover, miR-15a-5p could suppress hepatocellular carcinoma division and proliferation via targeting brain-derived neurotrophic factor (BDNF) (38). As indicated in the present study, miR-15a-5p was revealed to be underexpressed in EC, which indicated aggressive phenotypes and poor prognosis of EC patients. In addition, it was also verified that miR-15a-5p overexpression could inhibit EC progression both in vivo and in vitro. Data also revealed that miR-15a-5p could target VEGFA and thereby regulated its expression, suggesting that VEGFA was an essential regulator of miR-15a-5p-mediated functions in EC. It is well known that EMT and Wnt/ $\beta$-catenin signaling are crucial factors in EC progression, and the present study revealed that miR-15a-5p exerted its roles in EC via blocking EMT and Wnt/ß-catenin.

In conclusion, decreased miR-15a-5p expression was associated with poor prognosis and malignant clinicopathologic features of EC patients. Moreover, miR-15a-5p overexpression could downregulate VEGFA in EC cells, resulting in suppression of cell growth, invasion and migration. Additionally, it was also verified that miR-15a-5p could regulate EMT and the Wnt/ $\beta$-catenin pathway. The aforementioned data may provide novel insights into the identification of promising therapeutic and diagnostic strategies for EC treatment.

\section{Acknowledgements}

Not applicable.

\section{Funding}

No funding was received.

\section{Availability of data and materials}

The datasets used during the present study are available from the corresponding author upon reasonable request.

\section{Authors' contributions}

HW and QY made substantial contributions to the conception and design of the study. JL and WC performed all experiments. $\mathrm{XJ}$ collected and interpreted the data and performed the statistical analysis. YW performed the literature research, collected samples/clinical data, and wrote and revised the manuscript. All authors read and approved the final manuscript.

\section{Ethics approval and consent to participate}

Informed consent was obtained from the subjects for the use of their samples for experimentation. The present study conformed to the Code of Ethics from the Ethics Committee of Weifang People's Hospital (Weifang, China). Animal experiments were approved by the Animal Care and Use Committee of Weifang People's Hospital.

\section{Patient consent for publication}

Not applicable.

\section{Competing interests}

The authors declare that they have no competing interests.

\section{References}

1. Mahecha AM and Wang $\mathrm{H}$ : The influence of vascular endothelial grow th factor-A and matrix metalloproteinase-2 and -9 in angiogenesis, metastasis, and prognosis of endometrial cancer. Onco Targets Ther 10: 4617-4624, 2017.

2. Ray M and Fleming G: Management of advanced-stage and recurrent endometrial cancer. Semin Oncol 36: 145-154, 2009.

3. Davidson BA, Foote J, Clark LH, Broadwater G, Ehrisman J, Gehrig P, Graybill W, Alvarez Secord A and Havrilesky LJ: Tumor grade and chemotherapy response in endometrioid endometrial cancer. Gynecol Oncol Rep 17: 3-6, 2016.

4. Chen HX, Xu XX, Tan BZ, Zhang Z and Zhou XD: MicroRNA-29b inhibits angiogenesis by targeting VEGFA through the MAPK/ERK and PI3K/Akt signaling pathways in endometrial carcinoma. Cell Physiol Biochem 41: 933-946, 2017.

5. Pasquinelli AE: MicroRNAs and their targets: Recognition, regulation and an emerging reciprocal relationship. Nat Rev Genet 13: 271-282, 2012.

6. Krist B, Florczyk U, Pietraszek-Gremplewicz K, Jozkowicz A and Dulak J: The Role of miR-378a in metabolism, angiogenesis, and muscle biology. Int J Endocrinol 2015: 281756, 2015.

7. Srivastava AK, Banerjee A, Cui T, Han C, Cai S, Liu L, Wu D, Cui R, Li Z, Zhang X, et al: Inhibition of miR-328-3p impairs cancer stem cell function and prevents metastasis in ovarian cancer. Cancer Res 79: 2314-2326, 2019.

8. Kouri FM, Hurley LA, Daniel WL, Day ES, Hua Y, Hao L, Peng CY, Merkel TJ, Queisser MA, Ritner C, et al: miR-182 integrates apoptosis, growth, and differentiation programs in glioblastoma. Genes Dev 29: 732-745, 2015.

9. Zhang FB, Du Y, Tian Y, Ji ZG and Yang PQ: miR-1299 functions as a tumor suppressor to inhibit the proliferation and metastasis of prostate cancer by targeting NEK2. Eur Rev Med Pharmacol Sci 23: 530-538, 2019.

10. Peng H, Wang L, Su Q, Yi K, Du J and Wang Z: miR-31-5p promotes the cell growth, migration and invasion of colorectal cancer cells by targeting NUMB. Biomed Pharmacother 109: 208-216, 2019.

11. Zhen Z, Dong F, Shen H, Wang QG, Yang L and Hu J: miR-524 inhibits cell proliferation and induces cell apoptosis in thyroid cancer via targeting SPAG9. Eur Rev Med Pharmacol Sci 22: 3812-3818, 2018.

12. Zheng X, Xu K, Zhu L, Mao M, Zhang F and Cui L: miR-486-5p Act as a biomarker in endometrial carcinoma: Promotes cell proliferation, migration, invasion by targeting MARK1. Onco Targets Ther 13: 4843-4853, 2020.

13. Wang C and Liu B: miR-101-3p induces autophagy in endometrial carcinoma cells by targeting EZH2. Arch Gynecol Obstet 297: 1539-1548, 2018.

14. Zhang HC, Han YY, Zhang XM, Xiao N, Jiang T, Zhu S, Wang EP and Chen CB: miR-522 facilitates the prosperities of endometrial carcinoma cells by directly binding to monoamine oxidase B. Kaohsiung J Med Sci 35: 598-606, 2019.

15. Sales CB, Buim ME, de Souza RO, de Faro Valverde L, Mathias Machado MC, Reis MG, Soares FA, Ramos EA and Gurgel Rocha CA: Elevated VEGFA mRNA levels in oral squamous cell carcinomas and tumor margins: A preliminary study. J Oral Pathol Med 45: 481-485, 2016.

16. Claesson-Welsh L and Welsh M: VEGFA and tumour angiogenesis. J Intern Med 273: 114-127, 2013.

17. Peng T, Li Z, Li D and Wang S: MACC1 promotes angiogenesis in cholangiocarcinoma by upregulating VEGFA. Onco Targets Ther 12: 1893-1903, 2019.

18. Horwitz E, Stein I, Andreozzi M, Nemeth J, Shoham A, Pappo O, Schweitzer N, Tornillo L, Kanarek N, Quagliata L, et al: Human and mouse VEGFA-amplified hepatocellular carcinomas are highly sensitive to sorafenib treatment. Cancer Discov 4: 730-743, 2014. 
19. Luo $X$ and Feng GS: VEGFA genomic amplification tailors treatment of HCCs with sorafenib. Cancer Discov 4: 640-641, 2014.

20. Chen X, Xu X, Pan B, Zeng K, Xu M, Liu X, He B, Pan Y, Sun H and Wang S: miR-150-5p suppresses tumor progression by targeting VEGFA in colorectal cancer. Aging (Albany NY) 10: 3421-3437, 2018

21. Guset G, Costi S, Lazar E, Dema A, Cornianu M, Vernic C and Păiusan L: Expression of vascular endothelial growth factor (VEGF) and assessment of microvascular density with CD34 as prognostic markers for endometrial carcinoma. Rom J Morphol Embryol 51: 677-682, 2010.

22. Panda H, Pelakh L, Chuang TD, Luo X, Bukulmez O and Chegini N: Endometrial miR-200c is altered during transformation into cancerous states and targets the expression of ZEBs, VEGFA, FLT1, IKK $\beta$, KLF9, and FBLN5. Reprod Sci 19: 786-796, 2012.

23. Livak KJ and Schmittgen TD: Analysis of relative gene expression data using real-time quantitative PCR and the 2(-Delta Delta C(T)) method. Methods 25: 402-408, 2001.

24. Garcia DM, Baek D, Shin C, Bell GW, Grimson A and Bartel DP Weak seed-pairing stability and high target-site abundance decrease the proficiency of 1sy- 6 and other microRNAs. Nat Struct Mol Biol 18: 1139-1146, 2011.

25. Grimson A, Farh KK, Johnston WK, Garrett-Engele P, Lim LP and Bartel DP: MicroRNA targeting specificity in mammals: Determinants beyond seed pairing. Mol Cell 27: 91-105, 2007.

26. SGO Clinical Practice Endometrial Cancer Working Group; Burke WM, Orr J, Leitao M, Salom E, Gehrig P, Olawaiye AB, Brewer M, Boruta D, Villella J, et al: Endometrial cancer: A review and current management strategies: Part I. Gynecol Oncol 134: 385-392, 2014.

27. Yang CH, Zhang XY, Zhou LN, Wan Y, Song LL, Gu WL, Liu R, Ma YN, Meng HR, Tian YL and Zhang Y: LncRNA SNHG8 participates in the development of endometrial carcinoma through regulating c-MET expression by miR-152. Eur Rev Med Pharmacol Sci 22: 1629-1637, 2018.

28. Teague EM, Print CG and Hull ML: The role of microRNAs in endometriosis and associated reproductive conditions. Hum Reprod Update 16: 142-165, 2010.

29. Lamouille S, Connolly E, Smyth JW, Akhurst RJ and Derynck R: TGF- $\beta$-induced activation of mTOR complex 2 drives epithelial-mesenchymal transition and cell invasion. J Cell Sci 125: $1259-1273,2012$
30. Bullock MD, Sayan AE, Packham GK and Mirnezami AH MicroRNAs: Critical regulators of epithelial to mesenchymal (EMT) and mesenchymal to epithelial transition (MET) in cancer progression. Biol Cell 104: 3-12, 2012.

31. Nusse $R$ and Clevers $H$ : Wnt/beta-catenin signaling, disease, and emerging therapeutic modalities. Cell 169: 985-999, 2017.

32. Majidinia M, Aghazadeh J, Jahanban-Esfahlani R and Yousefi B: The roles of Wnt/beta-catenin pathway in tissue development and regenerative medicine. J Cell Physiol 233: 5598-5612, 2018.

33. Shen YN, He HG, Shi Y, Cao J, Yuan JY, Wang ZC, Shi CF, Zhu N, Wei YP, Liu F, et al: Kruppel-like factor 8 promotes cancer stem cell-like traits in hepatocellular carcinoma through Wnt/ $\beta$-catenin signaling. Mol Carcinog 56: 751-760, 2017.

34. Shen Q, He T and Yuan H: Hsa_circ_0002577 promotes endometrial carcinoma progression via regulating miR-197/CTNND1 axis and activating Wnt/ $\beta$-catenin pathway. Cell Cycle 18: 1229-1240, 2019.

35. Park SA, Kim LK, Kim YT, Heo TH and Kim HJ: Long non-coding RNA steroid receptor activator promotes the progression of endometrial cancer via Wnt/ $\beta$-catenin signaling pathway. Int J Biol Sci 16: 99-115, 2020.

36. Chen P, Xing T, Wang Q, Liu A, Liu H, Hu Y, Ji Y, Song Y and Wang D: MicroRNA-202 inhibits cell migration and invasion through targeting FGF2 and inactivating Wnt/ $\beta$-catenin signaling in endometrial carcinoma. Biosci Rep 39: BSR20190680, 2019.

37. Kontos CK, Tsiakanikas P, Avgeris M, Papadopoulos IN and Scorilas A: miR-15a-5p, A novel prognostic biomarker, predicting recurrent colorectal adenocarcinoma. Mol Diagn Ther 21: 453-464, 2017.

38. Long J, Jiang C, Liu B, Fang S and Kuang M: MicroRNA-15a-5p suppresses cancer proliferation and division in human hepatocellular carcinoma by targeting BDNF. Tumour Biol 37: 5821-5828, 2016.

This work is licensed under a Creative Commons Attribution-NonCommercial-NoDerivatives 4.0 International (CC BY-NC-ND 4.0) License. 